\title{
Exploiting inflammation for therapeutic gain in pancreatic cancer
}

\author{
C W Steele ${ }^{1,2}$, N B Jamieson ${ }^{2}$, T R J Evans ${ }^{1,3}$, C J McKay ${ }^{2}$, O J Sansom ${ }^{1}$, J P Morton ${ }^{1}$ and C R Carter ${ }^{*, 2}$ \\ ${ }^{1}$ The Beatson Institute for Cancer Research, Glasgow G61 1BD, UK; ${ }^{2}$ Department of Surgery, West of Scotland Pancreatic Unit, \\ Glasgow Royal Infirmary, Glasgow G4 OSF, UK and Institute of Cancer Sciences, College of Medical, Veterinary and Life Sciences, \\ University of Glasgow, Glasgow G61 1BD, UK
}

Pancreatic ductal adenocarcinoma (PDAC) is an aggressive malignancy associated with $<5 \% 5$-year survival, in which standard chemotherapeutics have limited benefit. The disease is associated with significant intra- and peritumoral inflammation and failure of protective immunosurveillance. Indeed, inflammatory signals are implicated in both tumour initiation and tumour progression. The major pathways regulating PDAC-associated inflammation are now being explored. Activation of leukocytes, and upregulation of cytokine and chemokine signalling pathways, both have been shown to modulate PDAC progression. Therefore, targeting inflammatory pathways may be of benefit as part of a multi-target approach to PDAC therapy. This review explores the pathways known to modulate inflammation at different stages of tumour development, drawing conclusions on their potential as therapeutic targets in PDAC.

Pancreatic ductal adenocarcinoma (PDAC) is one of the most lethal and aggressive of all malignancies, and as the 5th most common cause of cancer death in the United Kingdom represents a significant therapeutic challenge. Patients with potentially resectable disease are in the minority (10-15\%) because of extensive local spread or metastatic disease at presentation (Sener et al, 1999). Despite surgery, the 5-year survival rate is a dismal $3-5 \%$ due to secondary recurrence.

The European Study of Pancreatic and Ampullary Cancer (ESPAC) trials have consistently demonstrated a modest survival benefit associated with post-operative adjuvant therapy, with fluorouracil and gemcitabine equally effective (Neoptolemos et al, 2010). Unfortunately, although a subset of patients who receive gemcitabine have tumours responsive to these drugs, a significant number develop resistance (Liss and Thayer, 2012). Despite similar presentation and histological appearance, these tumours are biologically diverse and exhibit complex molecular and cellular heterogeneity, however, aggressive progression is common to all (Vincent et al, 2011). Indeed, recent elegant lineage tracing studies in mice have identified that cells may disseminate from pancreatic lesions even before the primary tumour is histologically evident, leading to a call for new approaches to PDAC therapy (Rhim et al, 2012; Tuveson and Neoptolemos, 2012). Management algorithms for PDAC must change, taking into account tumour biology, and stratifying therapy on an individual patient basis while pursuing strategies to combat recurrence in patients following surgical resection. The dependence of PDAC on certain crucial inflammatory mediators suggests that targeting of these central roles may hold hope for effective therapies.

The following review focuses on the failure of tumour immunosurveillance mechanisms in PDAC initiation and the accumulation in the pancreas of immunosuppressive cells that permits tumour progression, and discusses how failure of immunosuppression may be addressed therapeutically. We describe how tissue damage combined with oncogene activation (KRAS), loss of tumour suppressor genes (TSGs) (TP53 and SMAD4), deregulation of chemokines (CXCR2 ligands) and cytokines (interleukin (IL)-6, IL-1 $\alpha$ ), activation of downstream effectors (STAT3, NF- $\kappa \mathrm{B}$ ), and concurrent leukocyte recruitment by the tumour are key events in both initiation and progression of PDAC. Throughout, we consider the potential of these inflammatory mediators as therapeutic targets in PDAC taking into account the results of preclinical and clinical research to date.

\section{IMMUNE INFILTRATION DURING PDAC INITIATION AND} PROGRESSION

In healthy individuals, the adaptive immune system is responsible for tumour immunosurveillance, through which the host can identify, mount a response to, and destroy tumour cells.

*Correspondence: CR Carter; E-mail: rosscarterno1@gmail.com

Received 24 July 2012; revised 19 November 2012; accepted 3 January 2013;

published online 5 February 2013

(c) 2013 Cancer Research UK. All rights reserved 0007-0920/13

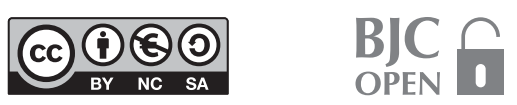



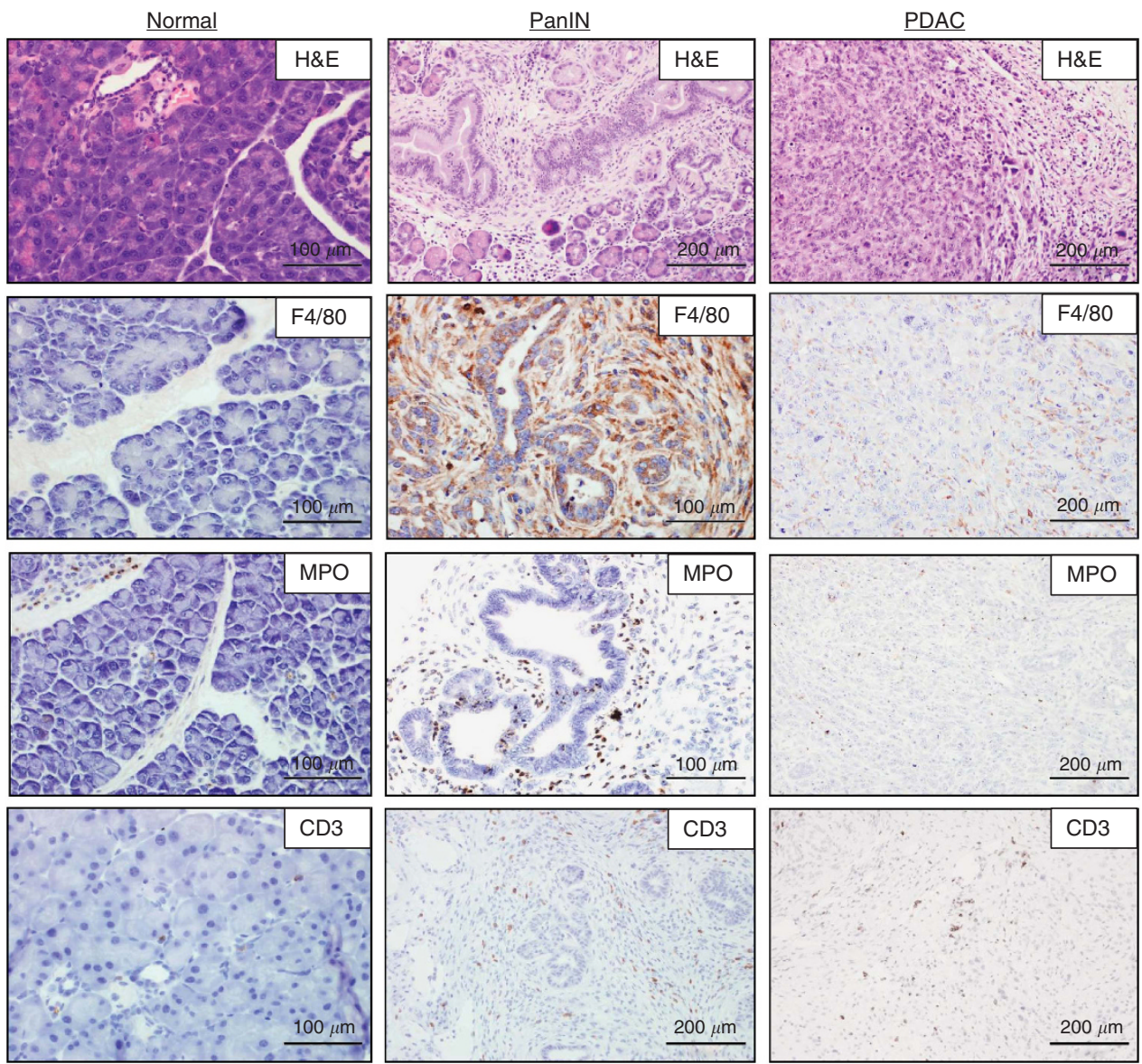

Figure 1. The role of inflammation in PDAC Initiation. PDAC evolution occurs from PanIN 1 through 3 to invasive PDAC with progressive accumulation of desmoplastic stroma (H\&E, top panels). The leukocyte infiltration surrounding lesions changes as PanINs progress.

Tumour-associated macrophage infiltration increases early in PDAC tumorigenesis as demonstrated by F4/80 immunohistochemistry (second row). Neutrophils (visualised by MPO staining) are abundant around developing PanIN lesions but sporadic within developed tumours (third row). Varying quantities of $\mathrm{T}$ cells are present within both PanINs and PDAC, as shown by CD3 immunohistochemistry (bottom row).

Tumorigenesis represents a failure of adaptive immunity, as in order for malignant transformation to progress unchecked tumour cells must escape this surveillance. The immune response to cancer is a dynamic process and can also initiate pathways that are in fact pro-tumorigenic. Even then a fine balance exists, as tumour progression is complex, reliant on interactions between the tumour and its activated microenvironment (Baumgart et al, 2011).

In early tumorigenesis, immune cells, including CD8 $+\mathrm{T}$ cells, detect danger signals from cancer cells and respond by eliminating these cells, leading to tumour immunogenicity. Dendritic cells are key regulators of tumour-specific immune responses in that they function as antigen presenting cells, activating CD8 + cytotoxic T lymphocyte (CTL) responses or stimulating CD $4+\mathrm{T}$ cells through interaction with MHC class II molecule-antigen complexes (Finn 2008). In pancreatic tumorigenesis, Hiraoka and colleagues reported that chemokine (C-X-C motif) ligand 17 (CXCL17) was responsible for infiltration of immature dendritic cells while the intercellular adhesion molecule (ICAM2) permitted killing of tumour cells by CD8 + - cells. During evolution from precursor lesions to PDAC they found that the host immune response was tempered and tumours developed immune tolerance by downregulating CXCL17 and ICAM2 (Hiraoka et al, 2011). Interestingly, Dillman and colleagues recently described the value of reintroducing dendritic cells loaded with antigen from autologous irradiated tumour cells in metastatic melanoma. Those patients who received this dendritic cell vaccine survived significantly longer than those irradiated with tumour cell vaccine alone (Dillman et al, 2011). Conversely, in PDAC, blockade of Toll-like receptor 4 (TLR4) signalling enhanced dendritic cell-mediated recruitment of T helper 2 (Th2) CD4 + cells, and this resulted in increased pancreatic inflammation and accelerated tumour progression (Ochi et al, 2012). Thus, depending on antigenic stimulus, dendritic cells can both promote and inhibit tumour progression.

Once tumour immune surveillance is overcome, the composition of the immune infiltrate changes and a pro-tumorigenic leukocyte profile emerges (Figure 1). It is not yet fully understood how these different pro and anti-tumoural components of the immune system are engaged, however, signals in the tumour microenvironment, including IL-10, can prevent dendritic cell activation, thus dampening the adaptive immune response (Koido et al, 2010). T cells can be both tumour suppressing and promoting depending upon their downstream target cells. CD4 + cells, particularly $\mathrm{T}$ regulatory cells and Th2 cells, increase in number over the course of pancreatic cancer progression, whereas CTL cells decrease (Clark et al, 2007). This dynamic circumstance leads to a progressive accumulation of immunosuppressive cells that inhibit the anti-tumoural immune response. In tumours infiltrated by $\mathrm{T}$ regulatory cells both innate and adaptive immunity may well be suppressed, while myeloid-derived suppressor cells (MDSCs) can also subdue anti-tumour activity (Gabrilovich and Nagaraj, 2009).

In this milieu of tumour cell killing and escape, the immune system must clear damaged tissue. Neutrophils move into tissues under the control of chemokine signalling, particularly CXCR2. Though short lived, neutrophils have the potential to induce significant tissue remodelling. Fridlender et al (2009) demonstrated that following transforming growth factor- $\beta$ (TGF- $\beta$ ) blockade, 
neutrophils exhibited an anti-tumour phenotype termed N1. Both neutrophils and MDSCs are Gr1+, CD11b + cells. The progressive accumulation of MDSCs in PDAC is widely recognised, while their immunosuppressive effect is clear. They are known to have the capacity to differentiate into both neutrophils and macrophages (Kusmartsev et al, 2005), and it has been suggested that pro-tumorigenic N2 neutrophils (TANs) may differentiate from MDSCs of splenic origin. Indeed two groups have demonstrated that in response to oncogenic Kras-dependent GM-CSF secretion, MDSCs are recruited to the tumour microenvironment where they have a role in suppressing CTL cell action and thus permitting tumorigenesis (Bayne et al, 2012; PylayevaGupta et al, 2012). When GM-CSF was suppressed, MDSCs failed to infiltrate the tumour microenvironment, and tumour growth was limited by infiltrating CTL cells. Furthermore, this effect was fully rescued by CTL depletion. Activated neutrophils also have the capacity to cause tissue damage via the release of matrix metalloproteinases (MMPs), while production of reactive oxygen species may promote mutagenesis. Reactive oxygen species have been implicated in the activation of $\mathrm{NF}-\kappa \mathrm{B}$, which has been intimately associated with the inflammatory response and PDAC progression (DeNicola et al, 2011).

Tumour-associated macrophages (TAMs) are present in early PDAC and persist throughout progression, representing an important component of the PDAC-associated leukocyte infiltrate (Clark et al, 2007). Intriguingly, TAMs can evolve to exhibit an M2 phenotype that is pro-tumorigenic in terms of promotion of growth and angiogenesis, and suppression of adaptive immunity (Sica and Mantovani, 2012). It is thought that this polarisation of macrophages from a tumour-suppressive M1 phenotype is initiated by cytokine signals, including IL-10 and TGF- $\beta$, received from T regulatory and tumour cells (Sica and Mantovani, 2012). This situation can be thought of as self-reinforcing, as production of pro-angiogenic molecules, including vascular endothelial growth factor (VEGF), attracts yet more macrophages to the tumour microenvironment, further enhancing angiogenesis. The plasticity of TAMs makes them an ideal therapeutic target for manipulation to generate an anti-tumour response.

Understanding the regulation of cancer immunosurveillance may help efforts to promote adaptive immune responses against PDAC, whereas the plasticity of TAMs and TANs may provide therapeutic opportunities for the promotion of an anti-tumour phenotype. There are examples where TAMs have been seen to elicit cancer-destructive properties. Hagemann et al (2008) have suggested that macrophages may be re-educated towards an antitumour function through control of NF- $\kappa \mathrm{B}$ activity. Triggering a 'good' innate immune response is a potential goal for anti-cancer therapy with the activation of M1 macrophages and inhibition of MDSCs and $\mathrm{T}$ regulatory cells as the important objectives.

Antibodies against M2 macrophages and regulatory T cells are now available for trial in preclinical models, while measures to harness the immune system to eradicate tumour cells are being considered. An excellent example was recently provided in a cohort of metastatic PDAC patients who received CD40-targeting monoclonal antibodies in addition to gemcitabine (Beatty et al, 2011). CD40 activation, somewhat unexpectedly, led to macrophage-dependent tumour regression. Promotion of properties of the adaptive immune system that protect us from malignancy in health holds promise for future trials.

\section{STROMAL CHANGES DURING TUMORIGENESIS}

In addition to immune cells other stromal elements have key roles in PDAC pathogenesis. Paracrine signalling through different molecules including TGF- $\beta$, VEGF, hepatocyte growth factor,
Sonic hedgehog (Shh), epidermal growth factor, fibroblast growth factors and insulin-like growth factors signal to the adjacent microenvironment, and in particular pancreatic stellate cells (PSCs). This interaction activates PSCs and is primarily responsible for fibrosis in PDAC (Neesse et al, 2011). Pancreatic stellate cells are also implicated in local tumour growth, in addition to their ability to travel to distant sites and support metastatic formation (Neesse et al, 2011). Furthermore, PSCs secrete factors into the microenvironment such as secreted protein acidic and rich in cysteine (SPARC), a protein which is associated with cell migration and wound healing, and high levels of which are associated with poor outcome in PDAC (Infante et al, 2007). Interestingly albumin-bound Paclitaxel (nab-paclitaxel) has been observed to bind SPARC-expressing fibroblasts, possibly providing a mechanism for targeting this specific cell type. Clinical trial of nabpaclitaxel in PDAC has shown some promise in combination with gemcitabine (Von Hoff et al, 2011), however, the full role of SPARC in modulating the tumour microenvironment is unclear, with few changes seen in tumour stroma when preclinical PDAC models were treated (Frese et al, 2012).

The dense, avascular, collagenous extracellular matrix that constitutes the majority of the tumour bulk in human PDAC has been shown to mechanically block the vascular delivery of chemotherapeutic agents to tumours in preclinical models (Neesse et al, 2011). It is anticipated that drugs that attack stromal elements responsible for tumour maintenance will be of clinical benefit. Thus far, clinical trials assessing anti-stromal therapies including anti-MMP and VEGF inhibitors such as Bevacizumab have proven disappointing (Neesse et al, 2011). Among others, Shh signalling through Smoothened (Smo) has been implicated in the coordination of tumour-stromal crosstalk in PDAC. The clinical trial based on the work of Olive et al (2009), who showed modest prolongation of survival of mice with PDAC following Smo inhibition in combination with gemcitabine, has been stopped due to better survival in the control arm (clinicaltrials.gov). In future, perhaps only trials demonstrating regression in primary tumour size and alteration of metastatic potential in murine models should be considered for trial in humans. However, there remains a belief that combating collagenous elements of PDAC stroma and promoting intra-tumoural vascularity will help delivery of standard chemotherapeutics to tumour cells. Tumours are dependent on both the protective effect of the stroma and interactions with stromal cells for progression. Hyaluronan has been identified as a key determinant of the stromal matrix with hyaluronidase treatment causing profound effects on tumour stroma in murine models of PDAC (Jacobetz et al, 2012; Provenzano et al, 2012). These drugs have also been shown to expand tumour vasculature and permit access of chemotherapeutics to the pancreas providing promise for ongoing clinical trials.

\section{THE IMPORTANCE OF INFLAMMATION IN PDAC} DEVELOPMENT

Mutations in the KRAS oncogene are presumed responsible for PDAC initiation and seen in the earliest PanIN lesions in nearly all cases. As these lesions progress from PanIN2 to PanIN3, mutations in the tumour suppressors TP53, CDKN2A, DPC4/SMAD4 and $B R C A 2$ are observed at varying frequencies, leading to invasive PDAC (Hruban et al, 2000). Recently, attention has switched to the elucidation of the role played by inflammation in the evolution of PDAC. Mouse models have been useful in demonstrating the importance of local inflammation in pancreatic tumorigenesis. The Barbacid lab has shown that experimentally induced chronic pancreatitis (CP) combined with targeted expression of oncogenic mutant $\mathrm{Kras}^{\mathrm{G} 12 \mathrm{~V}}$ to pancreatic acini, cooperate to induce PDAC formation (Guerra et al, 2007). Lee and Bar-Sagi (2010) 
demonstrated that on a background of inflammation, rather than activating senescence, Kras mutation led to decreased expression of Ink $4 a$ and a block in senescence, leading the authors to conclude that mutation of Kras in the presence of a pre-existing inflammation would lead rapidly to tumorigenesis. These studies suggest that PDAC can occur on a background of pre-existing, possibly subclinical, pancreatic inflammation. The identification of patients at risk of low-level pancreatic inflammation could allow clinical application of these findings in future.

Interestingly, patients with CP have an increased risk of PDAC, though the majority of patients do not develop cancer (Hezel et al, 2006). Patients presenting to clinics with $\mathrm{CP}$ represent the extreme end of the spectrum of pancreatic inflammation. Indeed, these patients have little functional pancreas as the majority is replaced with fibrous tissue (Kloppel 2007). Interestingly a high proportion of these patients carry KRAS mutations yet fail to develop PDAC, an observation that remains poorly explained (Rivera et al, 1997). Potentially, the mutational stress that low-grade pancreatic inflammation causes could create a more tumour-prone environment than the high-grade inflammation associated with CP.

Obesity, alcohol excess and particularly smoking have long been associated with low-grade systemic inflammation and predisposition to PDAC development, reviewed by Charkraborty et al (2011). Indeed in high-risk patient groups, including hereditary pancreatitis and familial PDAC families, smoking decreases the age of PDAC onset by an average of 20 years (Lowenfels et al, 1993). Although smoking is likely to result in an increased mutational burden, the tumour-promoting potential of the accompanying systemic inflammation should not be ignored. Indeed C-reactive protein (CRP), a marker of systemic inflammation, is a negative prognosticator in PDAC following resection, while systemic activation of $\mathrm{COX}-2, \mathrm{NF}-\kappa \mathrm{B}$, nitric oxide synthetase, production of IL-1, IL-6, IL- 8 and TNF- $\alpha$, and free radical oxygen formation have all been associated with PDAC progression (Farrow et al, 2004). Downregulation of systemic inflammation by generic antiinflammatories are already being trialled in other cancers. Epidemiological evidence does not conclusively support the use of aspirin or COX-2 inhibition in PDAC prevention (Tan et al, 2011), however, these widely available drugs may be of use in groups of PDAC patients with evidence of significant inflammation generated in response to their tumours, as measured by CRP. Inhibition of certain systemic mediators of inflammation has had some success in preclinical models. Pdx1-Cre Kras ${ }^{G 12 D}$ mice fed with COX-2 inhibitor showed a significant delay in PanIN progression (Funahashi et al, 2007). However, studies have failed to identify a role for COX inhibition in PDAC therapy. Despite initial promise, a phase II study of Celecoxib in addition to gemcitabine and cisplatin for advanced PDAC showed no benefit over chemotherapy alone (El-Rayes et al, 2005). In future trials, patients should be selected based upon COX-2 expression status to avoid missing potential therapeutic benefit.

\section{PDAC-ASSOCIATED MUTATIONS SUSTAIN AN} INFLAMMATORY MICROENVIRONMENT

The mounting of a response to tumour threat or injury is not the only way in which the immune system can become pathologically activated in PDAC. Initiating KRAS mutations induce upregulation of inflammatory pathways in PDAC, and downstream of KRAS the RAF signalling pathway induces production of chemokines and cytokines that may in themselves be pro-tumorigenic, including chemokines CXCL1 and CXCL8, the major ligands for CXCR2 (Balkwill, 2012). The oncogenic transcription factor c-Myc, also activated in tumours, promotes cell-autonomous proliferation and is key to tumour microenvironment remodelling via stimulation of inflammatory cells and cytokine production (Balkwill, 2012).
Ochi et al recently showed high expression of Toll-like receptor 7 (TLR7) in PDAC. They elegantly demonstrated in a murine model that TLR7 ligation accelerated pancreatic tumorigenesis via upregulation of inflammatory cascades including STAT3 and NF$\kappa \mathrm{B}$ pathways. Toll-like receptor 7 blockade protected against carcinogenesis and when mice lacking TLR7 exclusively within their inflammatory cells were generated they were completely protected from carcinogenesis (Ochi et al, 2012). Toll-like receptor 7 inhibition may have a role in combating the tumour microenvironment in PDAC by preventing signalling through downstream inflammatory cascades.

A number of upregulated inflammatory and oncogenic pathways in PDAC converge on the transcription factors STAT3 and $\mathrm{NF}-\kappa \mathrm{B}$, suggesting that these are excellent therapeutic targets. Two recent studies have demonstrated upregulation of STAT3 in PDAC (Fukuda et al, 2011; Lesina et al, 2011). Using mouse models of endogenous mutant Kras plus experimentally induced pancreatitis, the authors observed STAT3 activation in response to acinar damage. Activation was only sustained in the setting of Krasaccelerated metaplastic ducts and PanIN formation. When STAT3 was deleted, specifically within the pancreas, this significantly interfered with inflammation-mediated PanIN formation, and reduced both inflammatory cell infiltration $(\mathrm{CD} 45+$ cells $)$ and cytokine expression including IL-6. Candidate ligands for activation of STAT3 signalling in these model tumours were interrogated, and IL-6 was significantly upregulated and expressed predominantly by infiltrating macrophages. In human PDAC, serum IL-6 levels were significantly raised suggesting a potential link between systemic inflammation and PDAC tumorigenesis. Importantly IL-6 activation can occur via trans-signalling when IL-6 binds to a naturally occurring soluble form of IL-6R and forms a complex that induces IL-6-specific signalling in cells that lack a membrane-bound receptor. Indeed this latter form of signalling was found to be critical in the process of PDAC tumorigenesis. Furthermore Corcoran et al (2011) have established that pSTAT3 levels predict PDAC cell sensitivity to JAK2 inhibition, suggesting pSTAT3 status may be used in future to select patients for JAK/STAT inhibition.

Several studies have shown NF- $\kappa \mathrm{B}$ signalling to be an important signalling node in pancreatic cancer. In chronic inflammationassociated PDAC, NF- $\kappa$ B expression leads to secretion of the protumorigenic Shh ligand by macrophages (Yamasaki et al, 2010). When stimulated by cytokines such as TNF- $\alpha$, NF- $\kappa \mathrm{B}$ activates expression of growth-promoting molecules including cyclin D1 and E, c-Myc and IL-6. There is also evidence that mutant KRAS can activate NF- $\kappa \mathrm{B}$ through IL- $1 \alpha$ and p62. Meilisi et al identified autocrine IL- $1 \alpha$ secretion from PDAC cells to be the mechanism of constitutive NF- $\kappa \mathrm{B}$ activation. They showed that in response to IL- $1 \alpha$ stimulation, PDAC cells exhibited an invasive phenotype in vitro and when injected orthotopically into the murine pancreas generated liver metastases. This phenotype was reversed upon inhibition of NF- $\kappa \mathrm{B}$ (Melisi et al, 2009). Interestingly IKK2/ $\beta$, a kinase required to release NF- $\kappa \mathrm{B}$ from inhibition, is necessary for the induction of $\mathrm{CP}$, and PanIN and PDAC formation following Kras mutation (Ling, 2012). When genetically inactivated in a normally rapid mouse model of PDAC driven by mutant Kras and conditional deletion of $C d k n 2 a$, mice fail to develop PDAC over a 1 -year period. In addition, proliferation was significantly reduced in Pdx1-Cre; Kras ${ }^{G 12 D /+}$; IKK2/B $B^{f l / f l}$ animals compared with $P d x 1-$ $\mathrm{Cre}$; Kras ${ }^{\mathrm{G} 12 \mathrm{D} /+}$ controls. Further, the inflammatory infiltrate seen in Pdx1-Cre; Kras ${ }^{G 12 D /+}$ controls, which included T and B cells, macrophages and neutrophils, was not observed in $\mathrm{Pd} x \mathrm{l}-\mathrm{Cre}$; Kras $^{G 12 D /+} ; I K K 2 / B^{f l f l}$ animals. These observations provide further evidence supporting a role for inflammation in PDAC development and a critical role for NF- $\kappa \mathrm{B}$ in this process.

Thus, murine models have provided compelling evidence for the dependence of PDAC initiation on intrinsically generated 
inflammation. The activation of NF- $\kappa \mathrm{B}$ and STAT3 signalling by upregulated systemic cytokines including IL-6, IL- $1 \alpha$, and TNF- $\alpha$ identifies many of these molecules as excellent therapeutic targets. Anti-TNF- $\alpha$ therapies such as infliximab are well established in inflammatory conditions such as inflammatory bowel disease. Egberts et al (2008) demonstrated in orthotopic PDAC models that anti-TNF therapy reduced primary tumour size and metastases, however, these results have not been translated to clinical trials. Targeting IL-6, a recognised mediator of PDAC tumorigenesis, may be of therapeutic benefit. IL-6 monoclonal antibodies, Siltuximab and Tocilizumab, which bind to the soluble form of the IL- 6 receptor are available for trial and currently under assessment in ovarian cancer (clinicaltrials.gov). Furthermore, key nodes in the transmission of inflammatory signals such as STAT3, which is strongly implicated in PDAC tumorigenesis, can now be targeted with direct inhibitors. Recently triterpenoids and rexinoids demonstrated good efficacy in STAT3 binding, and prolonged survival in the Kras ${ }^{G 12 D} ; p 53^{f l /+}$ mouse model (Liby et al, 2010). These studies suggest that the repositioning of some antiinflammatory to treat pancreatic cancer may offer clinical benefit in the future.

COOPERATION BETWEEN TSG MUTATION AND CHEMOKINE SIGNALLING IN PDAC PROGRESSION

Recent studies support the deregulation of CXC chemokines in latestage PDAC. Mutation of p53 can drive migration and metastasis in
PDAC and this process was recently shown to be dependent on NF$\kappa \mathrm{B}$ signalling downstream of CXCR2 ligand upregulation ( $\mathrm{Li}$ et al, 2011). In addition, CXCL5, a ligand for CXCR2, was overexpressed in human PDAC and marked patients with poor outcome, advanced tumour stage and increased tumour size. Chemokine signalling may be critical in promoting invasion and metastasis in PDAC, especially downstream of mutations in TP53.

Chemokine signalling may also be deregulated by SMAD4 mutations, found in 50\% of PDAC cases (Rozenblum et al, 1997). SMAD4 is a TGF- $\beta$ signalling molecule, and its loss is thought to influence PDAC tumorigenesis through coordination of TGF- $\beta$ signalling in the tumour and stroma, as both fibroblasts and epithelial cells respond to TGF- $\beta$ in the tumour microenvironment (Ijichi et al, 2011). In a mutant Kras mouse model, Ijichi et al (2011) demonstrated that TGF- $\beta$ receptor II knockout resulted in aggressive PDAC that histologically recapitulated human disease. Secretion of CXCR2 specific chemokines, including CXCL1 and CXCL5, was significantly increased in these mice, and regulated by TGF- $\beta$ signalling and NF- $\kappa$ B. Interestingly, stromal fibroblasts also expressed significantly higher CXCR2 levels compared with epithelial cells. Treatment with a CXCR2 inhibitor over a short period improved survival and reduced microvessel density, supporting the theory that CXCR2 ligands are important in driving tumour progression following TSG mutation in PDAC.

These studies support the notion that pro-inflammatory pathways can be regulated by multiple different driver mutations in PDAC. The prognostic significance of deregulated chemokines and the functional role they have following TSG mutation, together

Targeting inflammation in the treatment of PDAC

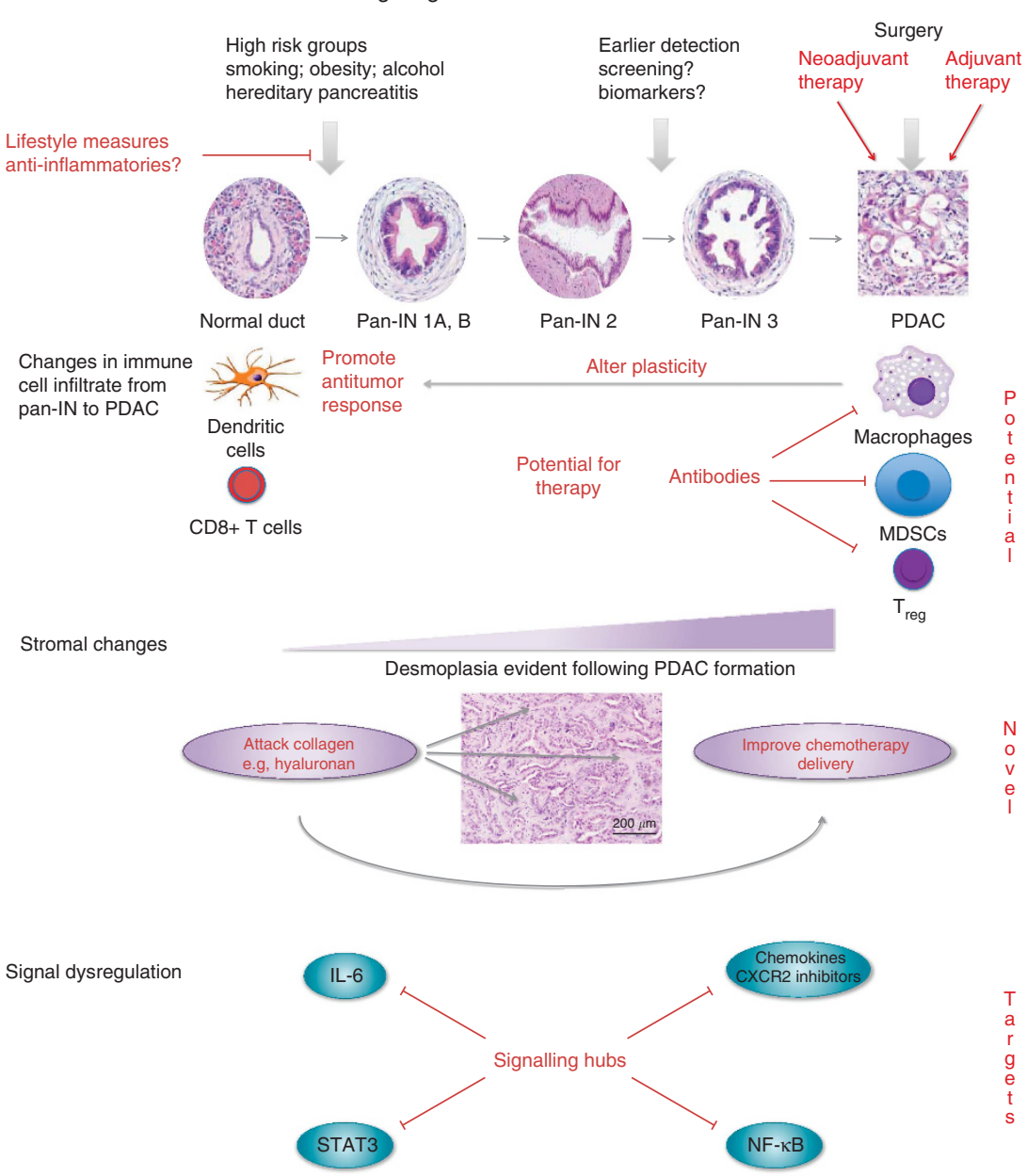

Figure 2. Targeting inflammation in the treatment of PDAC. Schematic illustrating progression to PDAC through PanIN stages, with potential therapeutic opportunities in both prevention and treatment of disease outlined. 
with the successful use of their inhibitors in other diseases, suggest that chemokines are potentially exciting therapeutic targets in patients with PDAC.

\section{METASTASIS AND INFLAMMATION}

Recent studies have shown metastases may occur in PDAC even before a primary tumour has formed, a behaviour associated with early epidermal mesenchymal transformation (Rhim et al, 2012). This process was accelerated in the presence of pancreatic inflammation, while the most invasive areas of tumour were seen at foci of inflammation. This phenotype was suppressed by dexamethasone, highlighting the integral role played by tumoural inflammation. CXCR2 has been shown to be an important pro-survival factor in metastatic breast cancer models (Acharyya et al, 2012). CXCR2 inhibition in this setting sensitised metastases to killing by standard chemotherapeutics. Chemokine-specific small molecules and monoclonal antibodies are undergoing preclinical study at present. The identification of inflammatory signalling pathways in the generation and maintenance of metastases in PDAC provides hope for combinatory anti-metastatic therapies in the near future.

\section{CONCLUSIONS}

Treating PDAC by targeting its reliance on the tumour microenvironment may be approached from three aspects (Figure 2): first by promotion of tumour-eliminating processes; second by suppression of tumour-promoting inflammation; and third via modulation of the protective fibrotic stroma of PDAC to allow access to tumour epithelium by conventional chemotherapeutics. The availability of preclinical in vivo models that accurately recapitulate the histological progression of PDAC and its complex microenvironment provides an excellent tool linking basic science with clinical application. The potential for preoperative characterisation of PDAC and direction of tumour-specific individualised therapy is within sight. Individualised multi-targeted therapy is likely to be necessary in order to treat PDAC effectively, as high recurrence rates following surgery and late presentation of disease remain significant hurdles.

Only $10-15 \%$ of PDAC are resectable at presentation highlighting that one of the greatest challenges facing PDAC management is earlier diagnosis. However, even those with R0 resection margins following surgery sadly suffer disease recurrence despite adjuvant therapy, often due to acquired drug resistance.

We have outlined the importance of the activated tumour microenvironment in PDAC maintenance and progression. Inflammation is implicated in the earliest stages of PDAC tumorigenesis, is strongly implicated in early metastases, and in tumour progression following TSG mutation. Combinatorial therapeutic regimens must look to capitalise on the importance of this tumour-microenvironment relationship. Aggressive targeted neoadjuvant therapy in surgically resectable patients should be considered, while those with more advanced disease may well respond to similar regimens. Incorporating newly developed antiinflammatory agents alongside standard chemotherapeutics may help provide much needed improvement in tumour sensitivity to therapy for patients suffering PDAC.

\section{ACKNOWLEDGEMENTS}

CS was funded by The Wellcome Trust while compiling this minireview. OS, JM and TRJE acknowledge the support of Cancer Research UK (http://info.cancerresearchuk.org/cancerstats/).

\section{REFERENCES}

Acharyya S, Oskarsson T, Vanharanta S, Malladi S, Kim J, Morris PG, Manova-Todorova K, Leversha M, Hogg N, Seshan VE, Norton L, Brogi E, Massague J (2012) A CXCL1 paracrine network links cancer chemoresistance and metastasis. Cell 150(1): 165-178.

Balkwill FR (2012) The chemokine system and cancer. J Pathol 226(2): $148-157$.

Baumgart S, Ellenrieder V, Fernandez-Zapico ME (2011) Oncogenic transcription factors: cornerstones of inflammation-linked pancreatic carcinogenesis. Gut 62(2): 310-316.

Bayne LJ, Beatty GL, Jhala N, Clark CE, Rhim AD, Stanger BZ, Vonderheide RH (2012) Tumor-derived granulocyte-macrophage colony-stimulating factor regulates myeloid inflammation and $\mathrm{T}$ cell immunity in pancreatic cancer. Cancer Cell 21(6): 822-835.

Beatty GL, Chiorean EG, Fishman MP, Saboury B, Teitelbaum UR, Sun W, Huhn RD, Song W, Li D, Sharp LL, Torigian DA, O’Dwyer PJ, Vonderheide RH (2011) CD40 agonists alter tumor stroma and show efficacy against pancreatic carcinoma in mice and humans. Science 331(6024): 1612-1616.

Chakraborty S, Baine MJ, Sasson AR, Batra SK (2011) Current status of molecular markers for early detection of sporadic pancreatic cancer. Biochim Biophys Acta 1815(1): 44-64.

Clark CE, Hingorani SR, Mick R, Combs C, Tuveson DA, Vonderheide RH (2007) Dynamics of the immune reaction to pancreatic cancer from inception to invasion. Cancer Res 67(19): 9518-9527.

Corcoran RB, Contino G, Deshpande V, Tzatsos A, Conrad C, Benes CH, Levy DE, Settleman J, Engelman JA, Bardeesy N (2011) STAT3 plays a critical role in KRAS-induced pancreatic tumorigenesis. Cancer Res 71(14): 5020-5029.

DeNicola GM, Karreth FA, Humpton TJ, Gopinathan A, Wei C, Frese K, Mangal D, Yu KH, Yeo CJ, Calhoun ES, Scrimieri F, Winter JM, Hruban RH, Iacobuzio-Donahue C, Kern SE, Blair IA, Tuveson DA (2011) Oncogene-induced Nrf2 transcription promotes ROS detoxification and tumorigenesis. Nature 475(7354): 106-109.

Dillman RO, Fogel GB, Cornforth AN, Selvan SR, Schiltz PM, DePriest C (2011) Features associated with survival in metastatic melanoma patients treated with patient-specific dendritic cell vaccines. Cancer Biother Radiopharm 26(4): 407-415.

Egberts JH, Cloosters V, Noack A, Schniewind B, Thon L, Klose S, Kettler B, von Forstner C, Kneitz C, Tepel J, Adam D, Wajant H, Kalthoff H, Trauzold A (2008) Anti-tumor necrosis factor therapy inhibits pancreatic tumor growth and metastasis. Cancer Res 68(5): 1443-1450.

El-Rayes BF, Zalupski MM, Shields AF, Ferris AM, Vaishampayan U, Heilbrun LK, Venkatramanamoorthy R, Adsay V, Philip PA (2005) A phase II study of celecoxib, gemcitabine, and cisplatin in advanced pancreatic cancer. Invest New Drugs 23(6): 583-590.

Farrow B, Sugiyama Y, Chen A, Uffort E, Nealon W, Mark Evers B (2004) Inflammatory mechanisms contributing to pancreatic cancer development. Ann Surg 239(6): 763-769discussion 769-771.

Finn OJ (2008) Cancer immunology. N Engl J Med 358(25): 2704-2715.

Frese KK, Neesse A, Cook N, Bapiro TE, Lolkema MP, Jodrell DI, Tuveson DA (2012) nab-Paclitaxel potentiates gemcitabine activity by reducing cytidine deaminase levels in a mouse model of pancreatic cancer. Cancer Discov 2(3): 260-269.

Fridlender ZG, Sun J, Kim S, Kapoor V, Cheng G, Ling L, Worthen GS, Albelda SM (2009) Polarization of tumor-associated neutrophil phenotype by TGF-beta: 'N1' versus 'N2' TAN. Cancer Cell 16(3): 183-194.

Fukuda A, Wang SC, Morris JPt, Folias AE, Liou A, Kim GE, Akira S, Boucher KM, Firpo MA, Mulvihill SJ, Hebrok M (2011) Stat3 and MMP7 contribute to pancreatic ductal adenocarcinoma initiation and progression. Cancer Cell 19(4): 441-455.

Funahashi H, Satake M, Dawson D, Huynh NA, Reber HA, Hines OJ, Eibl G (2007) Delayed progression of pancreatic intraepithelial neoplasia in a conditional Kras(G12D) mouse model by a selective cyclooxygenase- 2 inhibitor. Cancer Res 67(15): 7068-7071.

Gabrilovich DI, Nagaraj S (2009) Myeloid-derived suppressor cells as regulators of the immune system. Nat Rev Immunol 9(3): $162-174$.

Guerra C, Schuhmacher AJ, Canamero M, Grippo PJ, Verdaguer L, Perez-Gallego L, Dubus P, Sandgren EP, Barbacid M (2007) Chronic pancreatitis is essential for induction of pancreatic ductal adenocarcinoma by K-Ras oncogenes in adult mice. Cancer Cell 11(3): 291-302. 
Hagemann T, Lawrence T, McNeish I, Charles KA, Kulbe H, Thompson RG, Robinson SC, Balkwill FR (2008) 'Re-educating' tumor-associated macrophages by targeting NF-kappaB. J Exp Med 205(6): 1261-1268.

Hezel AF, Kimmelman AC, Stanger BZ, Bardeesy N, Depinho RA (2006) Genetics and biology of pancreatic ductal adenocarcinoma. Genes Dev 20(10): 1218-1249.

Hiraoka N, Yamazaki-Itoh R, Ino Y, Mizuguchi Y, Yamada T, Hirohashi S, Kanai Y (2011) CXCL17 and ICAM2 are associated with a potential antitumor immune response in early intraepithelial stages of human pancreatic carcinogenesis. Gastroenterology 140(1): 310-321.

Hruban RH, Goggins M, Parsons J, Kern SE (2000) Progression model for pancreatic cancer. Clin Cancer Res 6(8): 2969-2972.

Ijichi H, Chytil A, Gorska AE, Aakre ME, Bierie B, Tada M, Mohri D, Miyabayashi K, Asaoka Y, Maeda S, Ikenoue T, Tateishi K, Wright CV, Koike K, Omata M, Moses HL (2011) Inhibiting Cxcr2 disrupts tumorstromal interactions and improves survival in a mouse model of pancreatic ductal adenocarcinoma. J Clin Invest 121(10): 4106-4117.

Infante JR, Matsubayashi H, Sato N, Tonascia J, Klein AP, Riall TA, Yeo C, Iacobuzio-Donahue C, Goggins M (2007) Peritumoral fibroblast SPARC expression and patient outcome with resectable pancreatic adenocarcinoma. J Clin Oncol 25(3): 319-325.

Jacobetz MA, Chan DS, Neesse A, Bapiro TE, Cook N, Frese KK, Feig C, Nakagawa T, Caldwell ME, Zecchini HI, Lolkema MP, Jiang P, Kultti A, Thompson CB, Maneval DC, Jodrell DI, Frost GI, Shepard HM, Skepper JN, Tuveson DA (2012) Hyaluronan impairs vascular function and drug delivery in a mouse model of pancreatic cancer. Gut 62(1): 112-120.

Kloppel G (2007) Chronic pancreatitis, pseudotumors and other tumor-like lesions. Mod Pathol 20(Suppl 1): S113-S131.

Koido S, Homma S, Hara E, Namiki Y, Takahara A, Komita H, Nagasaki E, Ito M, Ohkusa T, Gong J, Tajiri H (2010) Regulation of tumor immunity by tumor/dendritic cell fusions. Clin Dev Immunol 2010: 516768 .

Kusmartsev S, Nagaraj S, Gabrilovich DI (2005) Tumor-associated CD8 + $\mathrm{T}$ cell tolerance induced by bone marrow-derived immature myeloid cells. J Immunol 175(7): 4583-4592.

Lee KE, Bar-Sagi D (2010) Oncogenic KRas suppresses inflammationassociated senescence of pancreatic ductal cells. Cancer Cell 18(5): $448-458$.

Lesina M, Kurkowski MU, Ludes K, Rose-John S, Treiber M, Kloppel G, Yoshimura A, Reindl W, Sipos B, Akira S, Schmid RM, Algul H (2011) Stat3/Socs 3 activation by IL-6 transsignaling promotes progression of pancreatic intraepithelial neoplasia and development of pancreatic cancer. Cancer Cell 19(4): 456-469.

Li A, King J, Moro A, Sugi MD, Dawson DW, Kaplan J, Li G, Lu X, Strieter RM, Burdick M, Go VL, Reber HA, Eibl G, Hines OJ (2011)

Overexpression of CXCL5 is associated with poor survival in patients with pancreatic cancer. Am J Pathol 178(3): 1340-1349.

Liby KT, Royce DB, Risingsong R, Williams CR, Maitra A, Hruban RH, Sporn MB (2010) Synthetic triterpenoids prolong survival in a transgenic mouse model of pancreatic cancer. Cancer Prev Res (Phila) 3(11): 1427-1434.

Ling Jea (2012) Kras G12D -induced IKK2/B/NFkB activation by IL-1alpha and p62 feedforward loops is requied for pancreatic ductal adenocarcinoma. Cancer Cell 21: 105-120.

Liss AS, Thayer SP (2012) Therapeutic targeting of pancreatic stroma. Trivandrum (India): Transworld Research Network. Chapter 9.

Lowenfels AB, Maisonneuve P, Cavallini G, Ammann RW, Lankisch PG, Andersen JR, Dimagno EP, Andren-Sandberg A, Domellof L (1993) Pancreatitis and the risk of pancreatic cancer. International Pancreatitis Study Group. N Engl J Med 328(20): 1433-1437.

Melisi D, Niu J, Chang Z, Xia Q, Peng B, Ishiyama S, Evans DB, Chiao PJ (2009) Secreted interleukin-1alpha induces a metastatic phenotype in pancreatic cancer by sustaining a constitutive activation of nuclear factorkappaB. Mol Cancer Res 7(5): 624-633.

Neesse A, Michl P, Frese KK, Feig C, Cook N, Jacobetz MA, Lolkema MP, Buchholz M, Olive KP, Gress TM, Tuveson DA (2011) Stromal biology and therapy in pancreatic cancer. Gut 60(6): 861-868.

Neoptolemos JP, Stocken DD, Bassi C, Ghaneh P, Cunningham D, Goldstein D, Padbury R, Moore MJ, Gallinger S, Mariette C, Wente MN, Izbicki JR, Friess H, Lerch MM, Dervenis C, Olah A, Butturini G, Doi R, Lind PA, Smith D, Valle JW, Palmer DH, Buckels JA, Thompson J, McKay CJ, Rawcliffe CL, Buchler MW (2010) Adjuvant chemotherapy with fluorouracil plus folinic acid vs gemcitabine following pancreatic cancer resection: a randomized controlled trial. JAMA 304(10): 1073-1081.

Ochi A, Graffeo CS, Zambirinis CP, Rehman A, Hackman M, Fallon N, Barilla RM, Henning JR, Jamal M, Rao R, Greco S, Deutsch M, Medina-Zea MV, Saeed UB, Ego-Osuala MO, Hajdu C, Miller G (2012) Toll-like receptor 7 regulates pancreatic carcinogenesis in mice and humans. J Clin Invest 122(11): 4118-4129.

Ochi A, Nguyen AH, Bedrosian AS, Mushlin HM, Zarbakhsh S, Barilla R, Zambirinis CP, Fallon NC, Rehman A, Pylayeva-Gupta Y, Badar S, Hajdu $\mathrm{CH}$, Frey AB, Bar-Sagi D, Miller G (2012) MyD88 inhibition amplifies dendritic cell capacity to promote pancreatic carcinogenesis via Th2 cells. J Exp Med 209(9): 1671-1687.

Olive KP, Jacobetz MA, Davidson CJ, Gopinathan A, McIntyre D, Honess D, Madhu B, Goldgraben MA, Caldwell ME, Allard D, Frese KK, Denicola G, Feig C, Combs C, Winter SP, Ireland-Zecchini H, Reichelt S, Howat WJ, Chang A, Dhara M, Wang L, Ruckert F, Grutzmann R, Pilarsky C, Izeradjene K, Hingorani SR, Huang P, Davies SE, Plunkett W, Egorin M, Hruban RH, Whitebread N, McGovern K, Adams J, Iacobuzio-Donahue C, Griffiths J, Tuveson DA (2009) Inhibition of Hedgehog signaling enhances delivery of chemotherapy in a mouse model of pancreatic cancer. Science 324(5933): 1457-1461.

Provenzano PP, Cuevas C, Chang AE, Goel VK, Von Hoff DD, Hingorani SR (2012) Enzymatic targeting of the stroma ablates physical barriers to treatment of pancreatic ductal adenocarcinoma. Cancer Cell 21(3): $418-429$.

Pylayeva-Gupta Y, Lee KE, Hajdu CH, Miller G, Bar-Sagi D (2012) Oncogenic Kras-induced GM-CSF production promotes the development of pancreatic neoplasia. Cancer Cell 21(6): 836-847.

Rhim AD, Mirek ET, Aiello NM, Maitra A, Bailey JM, McAllister F, Reichert M, Beatty GL, Rustgi AK, Vonderheide RH, Leach SD, Stanger BZ (2012) EMT and dissemination precede pancreatic tumor formation. Cell 148(1-2): 349-361.

Rivera JA, Rall CJ, Graeme-Cook F, Fernandez-del Castillo C, Shu P, Lakey N, Tepper R, Rattner DW, Warshaw AL, Rustgi AK (1997) Analysis of K-ras oncogene mutations in chronic pancreatitis with ductal hyperplasia. Surgery 121(1): 42-49.

Rozenblum E, Schutte M, Goggins M, Hahn SA, Panzer S, Zahurak M, Goodman SN, Sohn TA, Hruban RH, Yeo CJ, Kern SE (1997) Tumor-suppressive pathways in pancreatic carcinoma. Cancer Res 57(9): 1731-1734.

Sener SF, Fremgen A, Menck HR, Winchester DP (1999) Pancreatic cancer: a report of treatment and survival trends for 100,313 patients diagnosed from 1985-1995, using the National Cancer Database. J Am Coll Surg 189(1): $1-7$.

Sica A, Mantovani A (2012) Macrophage plasticity and polarization: in vivo veritas. J Clin Invest 122(3): 787-795.

Tan XL, Reid Lombardo KM, Bamlet WR, Oberg AL, Robinson DP, Anderson KE, Petersen GM (2011) Aspirin, nonsteroidal anti-inflammatory drugs, acetaminophen, and pancreatic cancer risk: a clinic-based case-control study. Cancer Prev Res (Phila) 4(11): 1835-1841.

Tuveson DA, Neoptolemos JP (2012) Understanding metastasis in pancreatic cancer: a call for new clinical approaches. Cell 148(1-2): 21-23.

Vincent A, Herman J, Schulick R, Hruban RH, Goggins M (2011) Pancreatic cancer. Lancet 378(9791): 607-620.

Von Hoff DD, Ramanathan RK, Borad MJ, Laheru DA, Smith LS, Wood TE, Korn RL, Desai N, Trieu V, Iglesias JL, Zhang H, Soon-Shiong P, Shi T, Rajeshkumar NV, Maitra A, Hidalgo M (2011) Gemcitabine plus nabpaclitaxel is an active regimen in patients with advanced pancreatic cancer: a phase I/II trial. J Clin Oncol 29(34): 4548-4554.

Yamasaki A, Kameda C, Xu R, Tanaka H, Tasaka T, Chikazawa N, Suzuki H, Morisaki T, Kubo M, Onishi H, Tanaka M, Katano M (2010) Nuclear factor kappaB-activated monocytes contribute to pancreatic cancer progression through the production of Sh. Cancer Immunol Immunother 59(5): 675-686.

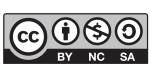
This work is licensed under the Creative Commons Attribution-NonCommercial-Share Alike 3.0 Unported License. To view a copy of this license, visit http://creativecommons. org/licenses/by-nc-sa/3.0/ 